\title{
Mapa de Rede de Impactos para Gestão Estratégica na Universidade
}

\author{
Naomar Monteiro de Almeida Filho' \\ Rogério Hermida Quintella' \\ Denise Maria Barreto Coutinho' \\ Francisco José Gomes Mesquita' \\ Osvaldo Barreto Filho'
}

'Universidade Federal da Bahia (UFBA), Salvador/BA - Brasil

RESUMO - Mapa de Rede de Impactos para Gestão Estratégica na Universidade. O trabalho introduz uma nova tecnologia de planejamento não linear, fundada na teoria da complexidade, a partir de um estudo de caso sobre sua aplicação em instituição universitária pública brasileira de grande porte. Apresenta os antecedentes da aplicação desta tecnologia de gestão no âmbito das organizações de produção de conhecimento e descreve as distintas escolas de pensamento estratégico-organizacional, enfocando suas bases lógicas. Na sequência, discute o caso da universidade, detalhando o uso da ferramenta Mapa de Rede de Impactos e os efeitos da utilização desta tecnologia em indicadores de desenvolvimento institucional. Na última parte, discute impactos e resultados alcançados.

Palavras-chave: Mapa de Rede de Impactos. Gestão Estratégica. Planejamento Não Linear. Estudos sobre Universidade.

ABSTRACT - Outcome Network Mapping for Strategic Management in the University. This paper introduces a new nonlinear planning technology based on complexity theory, with a case study of its application in a large Brazilian public university. We present the background of this management technology as applied to institutions of knowledge production. Furthermore, we describe different schools of organizational-strategic thinking, focusing on its logical bases. Following the presentation of the university case, we describe the use of the Outcome Network Mapping tool and its effects on indicators of institutional development. Finally, we discuss impacts and results.

Keywords: Outcome Network Map. Strategic Management. Nonlinear Planning. University Studies. 
Mapa de Rede de Impactos para Gestão Estratégica na Universidade

\section{Introdução}

Em 1990, Peter Senge escreve um texto clássico, intitulado A Quinta Disciplina, no qual introduz os conceitos de organização que aprende (learning organizations) e de aprendizagem em equipe (team learning), como fundamentos do pensamento sistêmico aplicado à gestão (Senge, 2010). Na perspectiva de uma ecologia das organizações complexas, esse autor e colaboradores desenvolvem o conceito de mudança para a sustentabilidade (Senge et al., 2005), aplicando-o particularmente à prospecção de contextos educacionais do futuro (Senge, 2012). Nessa abordagem, para concretizar projetos de mudança profunda e sustentável em instituições sociais de alta complexidade, recursos financeiros e insumos concretos são necessários, mas não suficientes. Nesse sentido, pelo menos dois movimentos mostram-se imprescindíveis: identificar e mobilizar os vetores políticos da organização e aplicar métodos avançados de gestão estratégica sistêmica, incluindo mapas mentais e processos educacionais (Senge, 2010). Trata-se, nesse caso, de garantir o aporte de investimentos imateriais sob a forma de novas tecnologias gerenciais orientadas para a dinamização política da organização e transformação sustentada da sua cultura institucional.

Abordagens contemporâneas de gestão, que vão desde a Workplace Democracy (Rayasam, 2008) até o Strategic Management (Niven, 2008), valorizam planejamento e mobilização, buscando engajamento e participação de atores e agentes em relação à eficiência e à sustentabilidade de resultados organizacionais e impactos sociais das intervenções. Nesse sentido, não se pode subestimar o papel das diversas escolas de pensamento estratégico, seus fundamentos, modelos teóricos, matrizes metodológicas, tecnologias, instrumentos e aplicações. Apesar disso, mesmo em suas versões mais atualizadas, na literatura especializada encontram-se raras indicações (Kiel, 2008; Earl; Carden; Smutylo, 2001) de métodos, tecnologias ou ferramentas de gestão capazes de subsidiar adequadamente o planejamento, a inovação ou o planejamento da inovação em instituições de conhecimento hipercomplexas como o são, por exemplo, as universidades federais brasileiras.

Nas últimas décadas, o campo de gestão das organizações complexas tem sido cada vez mais considerado na literatura de gestão estratégica. Nem sempre foi assim. Esse campo de conhecimento passou por quatro momentos distintos (Quintella, 1993). No primeiro momento, vigente até o final da década de 1950, sequer se utilizava o adjetivo estratégico ou o substantivo estratégia no jargão da gestão de organizações civis, relegando-os ao uso militar. No segundo momento, ao longo da década de 1960, a percepção do dinamismo dos mercados e a elevação dos então chamados níveis de turbulência ambiental exigiram abordagens mais dinâmicas e sistêmicas, levando ao surgimento de departamentos de business strategy nas escolas de administração. Paulatinamente, nas empresas de gestão mais avançada e no setor público, introduziram-se práticas de planejamento estratégico que poderíamos chamar de planejamento clássico. Não obstante, Igor Ansoff, ao escrever o clássico From 
Almeida Filho; Quintella; Coutinho; Mesquita; Barreto Filho

Strategic Planning to Strategic Management em 1976, já prenunciava as mudanças necessárias para um planejamento estratégico mais simples, rápido e de curto prazo. No momento seguinte, todas essas mudanças vieram a influenciar as práticas de gestão das organizações. Nesse novo conceito de gestão estratégica, além de tipicamente incorporar revisões anuais ao planejamento estratégico, as organizações passaram a se preocupar grandemente com seus desdobramentos no cotidiano. Em paralelo, ainda nesse terceiro momento, emerge o paradigma porteriano de planejamento estratégico.

Na sequência, as provocações inicialmente acadêmicas que levaram à quarta geração das tecnologias de planejamento e administração estratégica se difundem grandemente ao final dos anos 1990 e no início do século XXI. Essa nova forma de gerir estrategicamente o cotidiano das organizações se conforma com o trabalho de Kaplan e Norton e sua metodologia de Balanced Scorecard - BSC (Nivem, 2008). Nessa fase, destaca-se, também, a difusão da abordagem crítica de Mintzberg e colaboradores (Mintzberg, 1990; Mintzberg; Ahlstrand; Lampel, 1998). Ao desconstruir premissas cartesianas lineares, compartilhadas tanto pelo planejamento estratégico quanto pela administração estratégica, Mintzberg (1994) propõe, entre outras coisas, aplicar a recém-consagrada teoria da complexidade à gestão de organizações em estado de mudança.

Tomando como pressupostos as diferenças e as complementaridades de formas de pensamento e instrumentalização da gestão estratégica nas organizações, o presente trabalho tem como objetivo trazer à discussão uma nova tecnologia de planejamento não linear fundada na teoria da complexidade, a partir de um estudo de caso sobre sua aplicação numa instituição universitária pública de grande porte situada no Brasil.

Após esta breve introdução, a segunda parte deste texto apresenta os antecedentes da aplicação dessa tecnologia de gestão no âmbito das organizações de produção de conhecimento. Na terceira parte do artigo, descrevem-se as distintas escolas de pensamento estratégicoorganizacionais, focando suas bases lógicas para que se possa melhor contextualizar a metodologia de planejamento não linear aqui abordada. Na sequência, discute-se o caso da universidade estudada, onde são detalhados, respectivamente, o uso da ferramenta do Mapa de Impactos em Rede e o quadro de efeitos da utilização desta tecnologia em indicadores de desenvolvimento institucional. Por fim, na quinta e última parte do trabalho, discutem-se os impactos e resultados alcançados, além das considerações finais.

\section{Modelos de Planejamento/gestão Aplicados a Universidades}

Nas duas últimas décadas do século XX, técnicas de planejamento estratégico da perspectiva porteriana ${ }^{1}$ tornaram-se parte dominante dos modelos de gestão acadêmica, principalmente nos Estados Unidos. Uma verdadeira febre ocorreu nos planning offices das suas uni-

Educação \& Realidade, Porto Alegre, v. 39, n. 1, p. 277-301, jan./mar. 2014.

Disponível em: <http://www.ufrgs.br/edu_realidade> 
Mapa de Rede de Impactos para Gestão Estratégica na Universidade

versidades, preocupadas com eficiência num ambiente de redução do apoio governamental na perspectiva neoliberal de Reagan/Thatcher. Apresentações apologéticas das virtudes e aplicações do planejamento estratégico, como, por exemplo, as de Keller (1983), Rowley, Lujan e Dolence (1997) e Lerner (1999), contribuíram para a difusão dessas tecnologias na área. Em alguns casos, tais estratégias foram inscritas nos marcos estatutários das instituições de educação superior, como no caso da Universidade de Wisconsin (Paris, 2003).

No Brasil, a partir da redemocratização do país e da reestruturação constitucional do Estado, dispositivos normativos de gestão foram adotados inclusive pelos órgãos de controle para monitoramento e avaliação do serviço público. Instrumentos de fixação de diretrizes e metas, como o Projeto Político-Pedagógico (PPP) e o Plano de Desenvolvimento Institucional (PDI), permanecem como elementos residuais dessa abordagem.

Em paralelo, modelos de administração estratégica de base ansoffiana ${ }^{2}$ parecem ter voltado a dominar o cenário da gestão universitária, principalmente nos países da União Europeia. Um exemplo dessa tendência é o European Centre for the Strategic Management of Universities (ESMU), criado em 1986 para promover a gestão estratégica das universidades europeias com base no modelo do Strategic Management e que se mantém como importante centro difusor dessa perspectiva.

De forma geral, percebe-se que, desafiadas pelo Processo de Bolonha $^{3}$, as universidades europeias encontraram respostas imediatas nesse modelo de planejamento. Tavernier (2005) justifica tal necessidade a partir da generalizada incapacidade dos governos de se responsabilizarem por todo o custo da educação superior e, paralelamente, pelo que ele denomina Science Explosion. No Brasil, essa abordagem convencional tem dominado as poucas iniciativas de gestão estratégica das universidades. Um exemplo bastante ilustrativo é o Projeto Poli 2015, elaborado em 2003, que estabelecia todo um planejamento baseado na metodologia do Balanced Scorecard de Kaplan e Norton, para que a Escola Politécnica da USP alcançasse a sua Visão de Futuro para 2015 (Leão, 2007). A Figura 1 ilustra o caso.

Mais recentemente, Cao e Zhang (2009) propuseram elevar a outro nível a perspectiva inovadora da gestão estratégica de universidades, explicitando inclusive o que entendem ser a necessidade urgente de "gestão estratégica da ciência". Nesse sentido, a perspectiva do planejamento não linear, inspirada em Mintzberg e seguidores, encontra ressonância no contexto da crise contemporânea da educação superior. Bartnett (2000), em um importante artigo intitulado University Knowledge in an Age of Supercomplexity, chega a afirmar: "For various reasons, it is becoming felt that the knowledge function of the university is being undermined. Some, indeed, have come to suggest that we are witnessing 'the end of knowledge' in higher education"4.

O caráter hipercomplexo das organizações do tipo universidade é sobredeterminado por vários elementos, tais como: variação de porte institucional, pluralidade de campos de conhecimento, diversidade 
de modalidades de organização, baixo nível de hierarquização. Nesse último aspecto em particular, observa-se a grande mobilidade na hierarquia existente, com a peculiaridade da mudança de posição hierárquica do sujeito a depender da situação. Por exemplo, numa reunião de colegiado, o diretor da unidade se subordina ao coordenador do programa ou curso; um reitor pesquisador se subordina a outro gestor numa reunião de pós-graduação. Isso confere grande peso à subjetividade e às variáveis micropolíticas em processos decisórios e operacionais, acrescentando-lhes complexidade de gestão em grau elevado. É justamente nesse conjunto de variáveis hierárquicas e políticas, por exemplo, que se pode encontrar marcante distinção entre as universidades brasileiras e a maioria de suas congêneres internacionais mais renomadas.

Não obstante, em geral são poucas as iniciativas de interpretar o contexto acadêmico e a instituição universitária numa perspectiva da complexidade como, por exemplo, a proposição mais analítica do que instrumental de Duke (1992), sem maiores repercussões posteriores. Recentemente, Munck e McConnell (2009) apresentam um modelo de análise de cenários, talvez único do ponto de vista comparativo, para integração do planejamento estratégico à gestão de uma instituição universitária na Irlanda.

Figura 1 - Exemplo de Aplicação da Metodologia do BSC à Escola Politécnica da USP

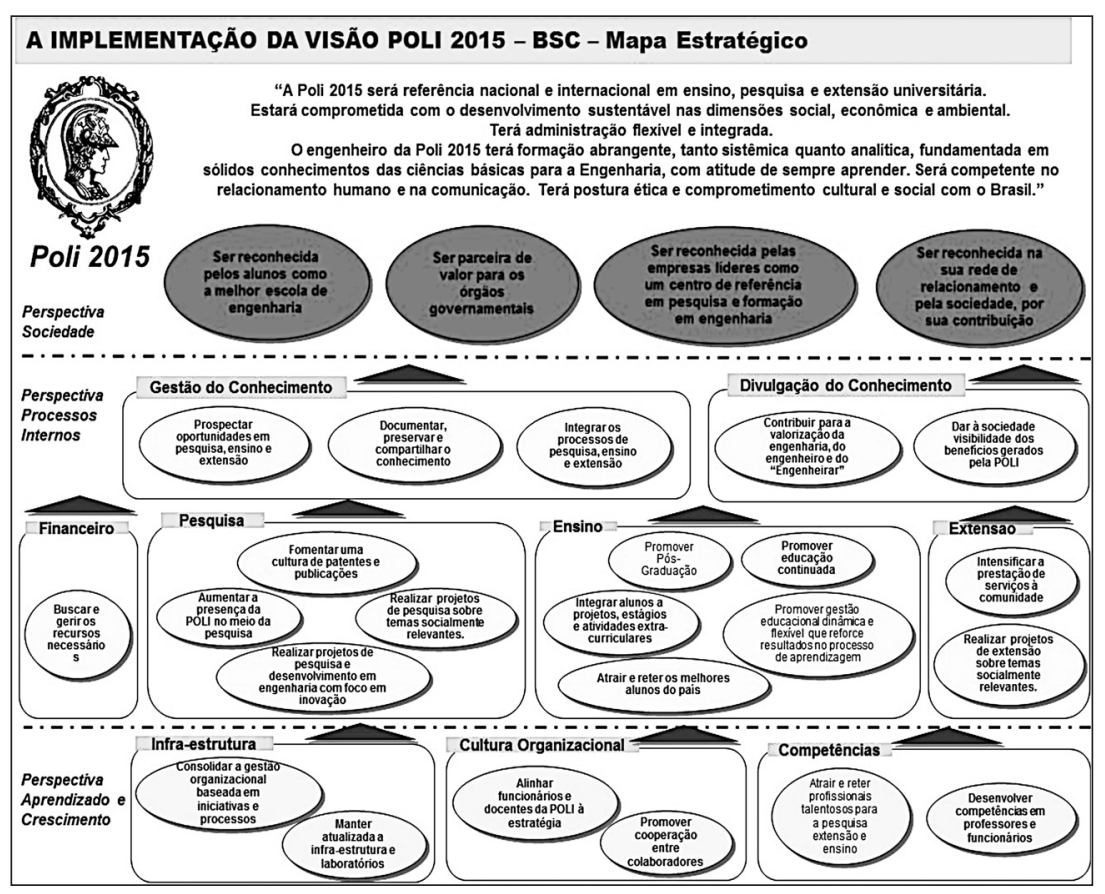

Fonte: Disponível em: <www.pcs.usp.br/ pcs0597/Poli\%202015\%20Sinatora.ppt>. Acesso em: 28 dez. 2013.

Educação \& Realidade, Porto Alegre, v. 39, n. 1, p. 277-301, jan./mar. 2014.

Disponível em: <http://www.ufrgs.br/edu_realidade> 
Mapa de Rede de Impactos para Gestão Estratégica na Universidade

As seminais contribuições teóricas e empíricas de Ansoff (1991), Porter (1998) e Mintzberg (1990) nasceram primordialmente no ambiente empresarial. Suas aplicações ao mundo acadêmico e, particularmente, às universidades públicas brasileiras, no entanto, não podem prescindir de considerações e ajustes a esta realidade tão diversa, principalmente quando se busca a transformação estrutural e a inovação na gestão dessas organizações. Para isso, é pertinente apresentar e discutir com mais cuidado a fundamentação teórica e metodológica das propostas de planejamento e gestão baseadas em modelos de complexidade (Patton, 2010).

\section{Escolas de Pensamento Estratégico-Organizacionais e o Planejamento Não Linear}

Mintzberg, Ahlstrand e Lampel (1998) avaliaram o estado da arte dos estudos de estratégia organizacional, a partir de extensa revisão de cerca de dois mil dos mais importantes trabalhos científicos publicados sobre o tema. Esse trabalho seminal permite uma visão sistêmica das distintas escolas de pensamento estratégico que compõem esse campo de conhecimento, ao tempo em que ajuda a explicar as diferenças entre elas, conformando, segundo Vasconcelos (2001), uma das mais ambiciosas classificações de estratégia já realizadas. O resultado daquele estudo é uma grade de classificação que agrupa a produção técnica e científica da área ao longo de 40 anos, em dez escolas de pensamento. Essas escolas de pensamento são caracterizadas conforme suas ideiaseixo, de forma aproximadamente cronológica, sendo resumidas a seguir a partir do trabalho de Quintella e Cabral (2007), com destaque para aquelas que melhor se coadunam com a metodologia de planejamento aqui abordada.

1) Escola do Design. Para esta escola, o principal papel do gestor é analítico, cabendo ao pensamento preceder a ação em busca de pontos fortes e fracos da organização, indicando riscos e oportunidades. Aborda também, embora com menor clareza e ênfase, os valores do gestor e sua responsabilidade social como influentes na escolha de tal estratégia. Nessa perspectiva, a concepção de estratégia recomenda simplicidade às organizações, entre outras razões, por limitar a responsabilidade de controle e formulação ao executivo principal.

2) Escola do Planejamento. Nessa escola, a gestão compreende um processo controlado, extremamente formal, que produz estratégias que devem ser integralmente explicitadas antes de implementadas. Planejadores profissionais são os principais condutores do processo.

3) Escola do Posicionamento. Aqui, o planejador profissional torna-se um analista que seleciona e recomenda estratégias ótimas aos gestores. Porter (1998) é sua referência central, instrumentalizando-a com as clássicas ferramentas de cadeia de valores e estratégias genéricas. 
4) Escola Empreendedora. Como a escola anterior, tem sua origem no campo da Economia; nesse caso, porém, o empreendedor tem papel proeminente. Diferentemente das anteriores, essa escola tem natureza puramente descritiva, buscando compreender o processo de formação de estratégia na medida em que ele se manifesta.

5) Escola da Cognição. Procura entender o funcionamento do cérebro humano para avaliar como, a partir da percepção da realidade, as estratégias se formam na mente do estrategista.

6) Escola do Aprendizado. Aqui, as estratégias emergem quando os atores, atuando individualmente ou na maioria das vezes coletivamente, aprendem sobre uma situação, desenvolvendo a capacidade de a organização lidar com ela. Suas premissas passam pelo aprendizado coletivo e emergente de forma deliberada, sendo os atores comprometidos com os resultados globais. Os padrões emergentes podem e devem ser internalizados à estratégia formal. Gerenciamento eficiente significa capacidade de manter a aprendizagem ao mesmo tempo em que se equilibram mudanças com continuidade, sabendo o que e quando mudar. Esse balanceamento se deve ao fato de que o aprendizado influencia a formação de estratégias organizacionais mais complexas. Devido à natureza complexa e imprevisível do ambiente de uma organização, o controle deliberado se torna impraticável. A formação de estratégias necessita assumir a forma de um processo de aprendizado no qual formulação e implementação se confundem. O papel da liderança, portanto, é o de conduzir o processo de aprendizado, no que se distingue da escola cognitiva mencionada anteriormente. Cyert e March (1963) e Prahalad e Hamel (1990) são referências nessa abordagem.

7) Escola do Poder. Explora a formação de estratégias como processo político de negociação e barganha. Existem duas vertentes nessa escola: a primeira é a do poder micro, que aborda tais relações sob a perspectiva de indivíduos e grupos internos à organização e considera relações políticas e de poder concentradas principalmente nos níveis diretivos das organizações. Já a vertente do poder macro baseia-se no poder das demandas externas (ambiente). Para os autores desta vertente, a organização busca seus interesses pelo controle ou pela cooperação com outras organizações através do uso de manobras estratégicas.

8) Escola Cultural. Para essa escola, a formação da estratégia constitui processo de interação social que, por aculturação, gera conhecimento tácito. A estratégia deriva, portanto, de intenções coletivas que podem se traduzir em barreiras ou facilitadores para determinadas escolhas. Essa escola vê a formação das estratégias como um processo deliberadamente emergente, trazendo a importante dimensão coletiva de um processo social. Nesse sentido, autores filiados à corrente da $V i$ são Baseada em Recursos (RBV) podem ser enquadrados no âmbito da escola cultural na medida em que se debruçam sobre as origens das capacidades dinâmicas na evolução da cultura organizacional.

Educação \& Realidade, Porto Alegre, v. 39, n. 1, p. 277-301, jan./mar. 2014. 283

Disponível em: <http://www.ufrgs.br/edu_realidade> 
Mapa de Rede de Impactos para Gestão Estratégica na Universidade

9) Escola Ambiental. Aqui, o ambiente organizacional ocupa papel preponderante no processo de formação estratégica, de modo a garantir uma adaptação adequada da organização.

10) Escola da Configuração. Nessa escola, para cada contexto, a organização deve adotar uma determinada forma de estrutura de formação de estratégias dependente dos seus ciclos de vida. Tais períodos (estáveis) alternam-se eventualmente com outras configurações (que podem ser explicadas, em cada momento, por uma das escolas anteriores).

Apesar das reconhecidas limitações, tipologias sistematizadoras podem ser úteis na visualização e compreensão dos problemas estratégicos de organizações hipercomplexas como as universidades brasileiras. A implantação do planejamento não linear, particularmente por meio do Mapa de Rede de Impactos (descrito em detalhe na seção seguinte), situa-se na interface entre a escola do aprendizado, a escola do poder e a escola cultural, uma vez que suas estratégias emergem por meio do aprendizado coletivo, em que os padrões emergentes são internalizados à estratégia formal, equilibrando mudanças com continuidade e formulação com implementação, tudo sob a coordenação da equipe de gestão, que, em seus processos de elaboração e implementação, emprega a negociação de divergências e a construção de consensos, com grande atenção à natureza política da interação social derivada de intenções coletivas em processos deliberadamente emergentes.

\section{Instrumentalizando o Planejamento Não Linear}

No modelo linear de planejamento, os diferentes objetivos da organização são tipicamente considerados como independentes ou apenas sequencialmente (ou linearmente) dependentes. Já no planejamento não linear, supõe-se que cada objetivo institucional interage com os demais, uma vez que as organizações constituem um sistema de processos, funções e metas interligados. Assim, o principal pressuposto do planejamento institucional não linear é que a instituição social constitui um "sistema adaptativo complexo" (Kiel, 1994, 2008). Sistemas desse tipo se caracterizam por propriedades especiais, como: emergência; coevolução; sistemas dentro de sistemas; iteração; variedade de requisitos; conectividade (relações entre agentes são geralmente mais importantes do que os próprios agentes); auto-organização (não há teleologia, mas constante reorganização para encontrar o melhor ajuste ao ambiente).

O planejamento institucional sistêmico, além de seu caráter de não linearidade, orienta-se pelo seguinte:

- defineo sistema combasenasinteraçõesentrepessoas, grupos, estruturaseideiaseascondutas, eventoseefeitosporelesproduzidos;

- identifica tendências internas para a auto-organização que orientam a emergência da ordem, direção e capacidade do sistema; 
Almeida Filho; Quintella; Coutinho; Mesquita; Barreto Filho

- focaliza processos mais do que estruturas ou efeitos;

- enfatiza a emergência como base da mudança resultante de infinitas interações entre elementos.

Instituições sociais são sistemas complexos que compreendem redes em transformação constante. Nesse sentido, justifica-se a explicitação dos conceitos articulados de mudança profunda (deep change) e de mudança sistêmica (systemic change). Tais conceitos foram introduzidos no início dos anos 1990 por Douglas Kiel, em Managing Chaos and Complexity in Government (1994), como alternativa à noção clássica de mudança controlada, incremental ou linear, focalizando a gestão estratégica de instituições públicas. Posteriormente, o conceito de mudança profunda foi apropriado por Quinn (1996), como base para uma teoria da liderança organizacional, e por Hammer (2004), para renovação estrutural de negócios ou empresas privadas.

Conforme Kiel (2008), as organizações, além de adaptativas e complexas, devem responder a um mundo em rápida mudança. Para tanto, sejam elas governamentais ou não, devem tornar-se instituições que aprendem através de mudanças profundas e sistêmicas. A transformação poderá produzir flutuações positivas que levam a estrutura organizacional a novos níveis de complexidade e ajuste adaptativo. A mudança profunda se refere à transição sustentada e sem retorno de elementos da estrutura ou dos sistemas de transformação dos processos fundamentais de uma instituição ou organização. A mudança sistêmica se dá através de pontos sensíveis, também chamados de nós críticos, muitas vezes inesperados em natureza, localização e efeito. Mudanças abruptas em sistemas complexos podem produzir efeitos projetados de curto prazo que, no longo prazo, não se traduzem em sustentabilidade ou consolidação das mudanças (Kiel, 2008).

Diversas estratégias metodológicas têm sido propostas para instrumentalizar e representar os sistemas de modo a permitir melhor acesso à sua lógica interna e ao entendimento da sua dinâmica geral. Dentre elas, destacam-se estratégias que representam conceitualmente os sistemas institucionais como redes complexas, formadas por sujeitos, órgãos e instâncias institucionais, programas e projetos. Kiel (2008) justifica a construção de imagens gráficas de redes complexas pela insuficiência das técnicas de exploração matemática disponíveis para fornecer os necessários fundamentos de análise. $\mathrm{O}$ uso das técnicas de análise visual tornou-se um elemento central dos estudos de complexidade. Dentre os vários métodos desse tipo disponíveis na literatura especializada, Waddell (2010) destaca:

- Social/Organizational/Inter-Organizational network analysis: Esta é a clássica análise de redes sociais aplicada especificamente ao entendimento das relações internas a uma organização ou entre organizações.

- Value Network Analysis (VNA): Os papéis-chave e os outputs em um sistema são definidos através de VNA, ajudando a mudar a

Educação \& Realidade, Porto Alegre, v. 39, n. 1, p. 277-301, jan./mar. 2014.

285

Disponível em: <http://www.ufrgs.br/edu_realidade> 
Mapa de Rede de Impactos para Gestão Estratégica na Universidade

mentalidade dos interessados em direção a uma perspectiva de análise de redes.

- Strategic Clarity Mapping (SCM): É voltado à geração de entendimento mútuo entre os diversos atores de uma rede sobre suas respectivas estratégias para a solução de uma questão, incluindo seus modelos mentais.

- Mind Mapping: Esta técnica mapeia as relações entre um conceito central e as palavras, ideias e tarefas que a ela se relacionam. Essa técnica é útil ao planejamento, à solução de problemas e à tomada de decisões ${ }^{5}$.

Uma importante derivação do mapeamento de redes que pretende sintetizar e articular diferentes grupos de métodos é a abordagem denominada developmental evaluation, de Patton (1994), apresentada como um conjunto articulado de princípios e procedimentos, concebidos para desenvolver medidas e mecanismos de monitoramento dos efeitos ou impactos (outcomes) à medida que emergem no sistema. Sua principal ferramenta metodológica é o Mapa de Rede de Impactos (Outcome Network Mapping). O Mapa de Rede de Impactos (MRI) compreende uma proposta de representação em rede não do sistema em si, mas da trama de iniciativas, interferências e influências visando à avaliação evolutiva da organização ou instituição (Earl; Carden; Smutylo, 2001; Smutylo, 2005).

Diferentemente da metodologia do Balanced Scorecard ${ }^{6}$ (Kaplan; Norton, 1992) e sua ferramenta Mapa Estratégico ${ }^{7}$ (Kaplan; Norton, 2004), dominantes no ambiente de mercado, a ferramenta aqui proposta, o MRI, pressupõe e enfatiza a não linearidade e a interação entre objetivos, podendo ser empregada tanto como dispositivo heurístico para exploração e compreensão da dinâmica das instituições sociais quanto como instrumento de planejamento estratégico de sua inovação institucional. Suas características lhe permitem hierarquizar programas, projetos e ações por seu potencial de gerar transformação no sistema como um todo.

As vantagens dessa nova forma de planejamento e gestão estratégica ficam patentes quando se observa, por exemplo, que na Figura 1 da seção anterior, onde o planejamento linear (mesmo baseado no BSC) leva a simplificações grosseiras, como ignorar os impactos da gestão financeira sobre a infraestrutura da organização, embora o fluxo oposto seja considerado. No planejamento não linear, todas as funções que conectam um objetivo (ou projeto) estratégico a outro são biunívocas e, na maioria dos casos, multívocas. Dessa maneira, podem-se considerar, na modelagem da rede-sistema, pontos sensíveis ou nós críticos e trajetórias de transformação, por exemplo, de uma instituição universitária tomada como caso de instituição hipercomplexa, apresentado a seguir. 


\section{Contexto e Caso}

O caso em questão neste estudo é uma instituição federal de conhecimento situada na Região Nordeste do Brasil com atribuições de ensino superior, pesquisa e extensão, doravante denominada Universidade ou, eventualmente, UFX. Entre 2002 e 2010, essa instituição foi objeto de dois períodos sucessivos de uma mesma gestão, diferenciados pela metodologia de planejamento adotada: o primeiro entre 20022006, caracterizado pelo uso de abordagens de planejamento linear; o segundo entre 2006-2010, quando se empregou um modelo de planejamento não linear para a gestão estratégica da instituição.

\section{Fase 1: Planejamento Estratégico Linear (2002-2006)}

No início do período contextualizado no estudo de caso, a Universidade tinha menos de 18 mil alunos em 55 cursos de graduação e pouco mais de 1.700 estudantes de pós-graduação matriculados em 44 mestrados e 17 doutorados. Seu corpo docente era composto por 1.900 professores, em sua maioria com título de mestre, enquanto seu orçamento montava a 586 milhões de reais.

Uma nova equipe assumiu a gestão da Universidade em agosto de 2002, realizando um esforço de saneamento financeiro e administrativo. Após a estruturação da equipe e iniciado o diagnóstico político da organização, realizou-se um processo tradicional de planejamento estratégico.

Nessa primeira fase do planejamento, princípios norteadores e eixos programáticos, ou temas estratégicos, foram sequenciados e desdobrados em matrizes de problematização, definindo situações-problema, estratégias, objetivos e metas. No que tange à missão e aos valores, num processo de consenso orientado, a equipe de gestão validou sua visão da instituição universitária como organização efetivamente comprometida com mudança, criação e inovação. A definição de temas e situações-problema permitiu discussão e estabelecimento de uma estratégia desdobrada em objetivos e projetos estratégicos.

Em decorrência do sucesso parcial do projeto proposto, a equipe de gestão da Universidade foi reconduzida em segundo mandato para o quadriênio 2006-2010. As ações que compuseram a nova pauta foram balizadas por dois eixos: (a) o redimensionamento institucional e político-acadêmico da instituição frente à política macro do país e (b) a renovação da capacidade de planejamento e gestão pela aplicação de técnicas de planejamento sistêmico não linear, a seguir descritas.

\section{Fase 2: Uso do Mapa de Rede de Impactos (2006-2010)}

No ano-base 2006, já se constatavam efeitos positivos da primeira etapa de gestão estratégica. Alguns dados descrevem o perfil institu- 
Mapa de Rede de Impactos para Gestão Estratégica na Universidade

cional nessa fase de transição: crescimento de 15\%, em relação a 2002, do número de matrículas na graduação; $43 \%$ de crescimento em pósgraduação; apenas $2,4 \%$ de incremento no orçamento executado, porém com saldo operacional positivo no fim do exercício.

Os Conselhos Superiores, desde a aprovação do Plano de Desenvolvimento Institucional (PDI) em 2004, haviam deliberado iniciar um processo de profunda revisão da sua política institucional a ser refletido em três eixos - estrutura, função e compromisso social -, visando a projetar o futuro da instituição. Durante o ano de 2005, algumas iniciativas foram tomadas nesse sentido, como a apresentação de estudos preliminares do Plano Diretor.

O movimento em defesa de uma nova arquitetura acadêmica para cursos de graduação no Brasil foi iniciado em 2006. Com o patrocínio da SESu/MEC, foram realizados eventos para divulgação e debate dessas ideias no meio universitário. O esgotamento do modelo profissional de graduação vigente no Brasil já então era uma constatação. Os estreitos campos de saber contemplados nos projetos pedagógicos, a precocidade na escolha das carreiras, os altos índices de evasão de alunos, o descompasso entre a rigidez da formação profissional, as amplas e diversificadas competências demandadas pelo mundo do trabalho e, sobretudo, os desafios educativos da sociedade do conhecimento constituíam fatores que exigiam um modelo de formação superior mais abrangente, flexível, integrador e de melhor qualidade.

O Programa de Reestruturação e Expansão das Universidades Federais (Reuni), vinculando significativa aplicação de recursos a propostas de renovação acadêmica ${ }^{8}$, veio ao encontro das expectativas das instituições federais que necessitavam de respaldo financeiro para se renovar tanto no plano infraestrutural quanto no organizacional e acadêmico. No advento do Reuni, a Universidade, portanto, encontrava-se num momento privilegiado, tanto em termos de conjuntura externa quanto de conjuntura interna, para consolidar, ampliar e aprofundar o processo de transformação já em curso.

As diretrizes do programa possibilitaram à Universidade elaborar uma proposta que, por um lado, contemplava elementos de inovação, como a criação de Bacharelados Interdisciplinares (BI), oferta de Cursos de Educação Superior Tecnológica (CEST) e retomada e ampliação do Programa de Licenciaturas Especiais (Prole); por outro lado, permitia a manutenção dos cursos existentes, com a devida articulação de suas estruturas curriculares a modelos reestruturados de arquitetura acadêmica (áreas de concentração oferecidas por unidades para permitir e incentivar a passagem aos cursos de progressão linear). A proposta de transformação da instituição universitária apresentada ao Reuni, que preenche critérios compatíveis com o modelo deep change, foi aprovada pela maioria das unidades de ensino que compunham a instituição em outubro de 2007. 
Na preparação para avançar no projeto de transformação institucional, a equipe já em 2006 considerava mais adequado o uso de técnicas de planejamento sistêmico não linear. Tal decisão foi tomada a partir da avaliação sistemática de um processo de transição institucional em curso, que revelava impactos positivos, porém limitados de recuperação da capacidade política e de gestão institucional e acadêmica. Dentro desse marco referencial inovador, foi possível construir uma matriz integrada de efeitos capaz de articular projetos e programas estratégicos necessários à transformação institucional proposta, como mostra, de maneira simplificada, o Quadro 1.

De forma a avançar, mantendo coerência com o processo executado nos primeiros quatro anos de reitorado, foi inicialmente montado um quadro de objetivos e metas estratégicas complementar ao que fora empregado anteriormente. Como pode ser observado nesse quadro, deixou-se de aplicar o conceito de temas estratégicos, passando-se a trabalhar com objetivos classificados de acordo com os quatro valores subjacentes à mudança desejada: renovação do ensino; valorização da pesquisa e inovação; compromisso social; e gestão participativa.

Para tanto, utilizou-se a metodologia de planejamento não linear apresentada na seção III. Como instrumento de planejamento estratégico, foi empregado como dispositivo heurístico o MRI, capaz de hierarquizar programas e projetos por seu potencial de gerar transformação no sistema como um todo. Segundo a extensiva pesquisa bibliográfica realizada em bases de pesquisa nacionais e internacionais (Scielo, Redalyc, Portal de Periódicos Capes, entre outros), trata-se da primeira tentativa de aplicação dessa nova metodologia de indução da inovação pela via do planejamento estratégico no contexto hipercomplexo de uma instituição universitária. Superando o modelo matricial de planejamento que organiza princípios e diretrizes em objetivos programáticos sequenciados e lineares, num quadro de efeitos previsíveis, as estratégias e ações componentes do plano são representadas por meio de uma rede hierarquizada, composta por pontos e nexos (links) formalizados sob um ponto de vista gráfico, na medida do seu impacto sobre a mudança do sistema.

Como se pode verificar na Figura 2, cada projeto ou programa tem como referencial gráfico circunferências de diâmetro variável, proporcional ao número de links por ele gerados e que o conectam a outros projetos. Programas de maior impacto, geradores de transformação nos outros elementos da rede, constituem nós críticos (hubs) a serem promovidos e cultivados. Isso se justifica plenamente, já que a meta não é a conservação e sim a introdução de mudança profunda (deep change) num sistema institucional aberto. 
Mapa de Rede de Impactos para Gestão Estratégica na Universidade

\section{Quadro 1 - Temas, Objetivos e Metas Estratégicas (2006-2010)}

\begin{tabular}{|c|c|c|c|}
\hline $\begin{array}{l}\text { Valores } \\
\text { subjacentes } \\
\text { àmudança } \\
\text { desejada }\end{array}$ & Objetivos estratégicos & $\begin{array}{l}\text { Principais metas } \\
\text { estratégicas }\end{array}$ & $\begin{array}{l}\text { Reali- } \\
\text { zação }\end{array}$ \\
\hline \multirow{9}{*}{$\begin{array}{l}\text { Renovação } \\
\text { do ensino }\end{array}$} & Implantação do Reuni & $\begin{array}{l}\text { Elaboração, aprovação } \\
\text { e financiamento }\end{array}$ & $\mathrm{S}$ \\
\hline & Implantação de cursos noturnos & $\begin{array}{l}\text { Elaboração, aprovação } \\
\text { efuncionamento }\end{array}$ & $\mathrm{S}$ \\
\hline & $\begin{array}{l}\text { Elaboração e implantação dos bacharelados } \\
\text { interdisciplinares }\end{array}$ & $\begin{array}{l}\text { Elaboração, aprovação } \\
\text { efuncionamento }\end{array}$ & $\mathrm{S}$ \\
\hline & Implantação de novos processos seletivos & $\begin{array}{l}\text { Elaboração, aprovação } \\
\text { e funcionamento }\end{array}$ & $\mathrm{S}$ \\
\hline & Implantação das novas ACCs* & $\begin{array}{l}\text { Elaboração, aprovação } \\
\text { efuncionamento }\end{array}$ & $\mathrm{S}$ \\
\hline & $\begin{array}{c}\text { Implantação de novos pavilhões de aulas multiu- } \\
\text { nidades }\end{array}$ & $\begin{array}{l}\text { Elaboração, aprovação } \\
\text { efuncionamento }\end{array}$ & $\mathrm{S}$ \\
\hline & $\begin{array}{c}\text { Criação e implantação do Instituto de Humanida- } \\
\text { des, Artes e Ciências (IHAC) }\end{array}$ & $\begin{array}{l}\text { Elaboração, aprovação } \\
\text { efuncionamento }\end{array}$ & $\mathrm{S}$ \\
\hline & $\begin{array}{c}\text { Criação e implantação do Programa de Renova- } \\
\text { ção Curricular } \\
\end{array}$ & $\begin{array}{l}\text { Elaboração, aprovação } \\
\text { efuncionamento }\end{array}$ & $\mathrm{N}$ \\
\hline & $\begin{array}{c}\text { Criação e implementação de novos mestrados } \\
\text { profissionais }\end{array}$ & Elaboração, aprovação & $\mathrm{N}$ \\
\hline \multirow{5}{*}{$\begin{array}{l}\text { Valorização } \\
\text { dapesquisa } \\
\text { einovação }\end{array}$} & FortalecimentodoNúcleodeInovaçãoTecnológica(NIT) & Reforma física & S \\
\hline & $\begin{array}{l}\text { Elaboração e implantação das novas Pró-Reitorias } \\
\text { de Pesquisa ede Pós-Graduação }\end{array}$ & Aprovação e operação & $\mathrm{S}$ \\
\hline & $\begin{array}{c}\text { Elevação do nível de internacionalização da } \\
\text { pesquisa }\end{array}$ & Fomento & $\mathrm{S}$ \\
\hline & Elaboração e implantação da UFXDigital & Elaboração & $\mathbf{N}$ \\
\hline & $\begin{array}{c}\text { Aprovação e implantação da TecnoUFXno Parque } \\
\text { Tecnológico situado na capital do estado }\end{array}$ & $\begin{array}{l}\text { Aprovação, financia- } \\
\text { mento }\end{array}$ & $\mathrm{S}$ \\
\hline \multirow{5}{*}{$\begin{array}{l}\text { Compromis- } \\
\text { so social }\end{array}$} & Mobilização e aprovação do Sistema de Museus & Aprovação e operação & $\mathrm{EP}$ \\
\hline & Criação e implantação da Nova Extensão & Sem expansão & $\mathbf{N}$ \\
\hline & Criação e aprovação da UFX Ecológica & $\begin{array}{c}\text { Elaboração, aprovação, } \\
\text { operação }\end{array}$ & EP \\
\hline & Criação da UFXPopular & Elaboração & $\mathbf{N}$ \\
\hline & Criação da UFXLivre & Elaboração & $\mathbf{N}$ \\
\hline \multirow{10}{*}{$\begin{array}{l}\text { Gestãopar- } \\
\text { ticipativa }\end{array}$} & \multirow[t]{2}{*}{ Novo Regimento e Estatuto } & $\begin{array}{c}\text { Elaboração e } \\
\text { aprovação do novo } \\
\text { Regimento Geral }\end{array}$ & $\mathrm{S}$ \\
\hline & & $\begin{array}{l}\text { Elaboração e aprova- } \\
\text { ção do novo Estatuto }\end{array}$ & S \\
\hline & ISAMCO & Reforma física & $\mathrm{EP}$ \\
\hline & Realizar a reforma da Assistência Estudantil & $\begin{array}{l}\text { Construção de resi- } \\
\text { dências }\end{array}$ & $\mathrm{S}$ \\
\hline & $\begin{array}{c}\text { Realizar a reforma do Programa de Apoio ao } \\
\text { Servidor (Pro-Servidor) }\end{array}$ & Elaboração & $\mathrm{N}$ \\
\hline & Desenvolver e modernizar o plano de saúde & Plano e implantação & $\mathrm{EP}$ \\
\hline & $\begin{array}{c}\text { Desenvolver e modernizar o Sistema UFXde } \\
\text { Saúde }\end{array}$ & $\begin{array}{l}\text { Construções, refor- } \\
\text { mas e mudanças }\end{array}$ & $\mathrm{EP}$ \\
\hline & $\begin{array}{l}\text { Ter um Plano de Desenvolvimento Institucional } \\
\text { que reflita a mudança buscada }\end{array}$ & $\begin{array}{l}\text { Elaboração e apro- } \\
\text { vação do novo Plano } \\
\text { de Desenvolvimento } \\
\text { Institucional (PDI) }\end{array}$ & $\mathrm{EP}$ \\
\hline & PlanoDiretorcoerentecomaestratégiadaUniversidade & Elaboraçãoeaprovação & $\mathrm{S}$ \\
\hline & Interiorizar a Universidade & $\begin{array}{c}\text { Implantação de } \\
\text { unidades no interior } \\
\text { do estado }\end{array}$ & $\mathrm{S}$ \\
\hline
\end{tabular}

Legendas: ACC - Atividades Curriculares em Comunidade; S - Sim, meta alcançada; $\mathrm{N}$ - Não, meta não alcançada; EP - Em processo. Fonte: elaborado pelos autores.

290 Educação \& Realidade, Porto Alegre, v. 39, n. 1, p. 277-301, jan./mar. 2014 Disponível em: <http://www.ufrgs.br/edu_realidade> 
O Mapa de Rede de Impactos (Figura 2), atualizado durante todo o percurso do segundo mandato, divide-se em quadrantes, equivalentes aos valores subjacentes à mudança desejada, definidas no Plano de Ação de 2006. Esses quadrantes são: renovação e integração do ensino de graduação e pós-graduação; valorização da pesquisa e inovação; reafirmação do compromisso social, redefinindo o conceito de extensão; modernização administrativa com gestão participativa. Note-se que os limites entre cada quadrante são representados por linhas pontilhadas, demarcando não fronteiras rígidas, mas sim áreas de superposição ou de transição. Programas que articulam funções designadas por dois ou mais quadrantes podem aí se posicionar, como, por exemplo, a priorização de novos mestrados profissionais, entre renovação do ensino e pesquisa e inovação.

A iniciativa que assumiu maior prioridade na gestão - o Plano Reuni-UFX - aumenta flagrantemente o peso relativo do quadrante ensino. Sua importância evidencia-se na riqueza e no volume de nexos indutores de transformação do sistema, repercutindo diretamente em 18 projetos ou programas (embora só 14 sejam ilustrados nessa versão simplificada). Em seguida, e a ele indiretamente vinculado, destaca-se a reforma do Regimento e do Estatuto. Em segundo nível de importância (por seus impactos cruzados) está a criação do Plano Diretor e, em seguida, os projetos UFX-Nova Pós-Graduação e Pesquisa. A constelação de efeitos do Plano Reuni-UFX na estrutura curricular, tais como flexibilização, mobilidade estudantil, Nova ACC, processos seletivos alternativos, cursos noturnos etc., realça a inovação prevista na implantação dos bacharelados interdisciplinares, vinculados à criação do Instituto de Humanidades, Artes e Ciências.

Além desses elementos inovadores constitutivos da rede de impactos, pode-se assinalar a centralidade da normalização estatutária e regimental da Universidade. Apesar de tecnicamente não poder ser alinhada como determinante da transformação e sim como efeito planejado do processo indutor, essa estratégia significa poderoso fator de consolidação e sustentabilidade das mudanças institucionais produzidas. Assim, é pertinente assinalar que a construção paciente e cuidadosa de uma conjuntura política interna, capaz de garantir pactos e consensos localizados, foi possível pelo sucesso na implantação do Plano Reuni nesta Universidade. De fato, isso propiciou a necessária credibilidade dos órgãos da administração central junto às instâncias deliberativas nos vários níveis de governança da instituição. Essa conjuntura política convergente permitiu a elaboração do novo marco normativo (Estatuto e Regimento Geral).

No outro extremo, pode-se ilustrar uma situação peculiar, o projeto Pró-Servidor. Não obstante o seu enorme potencial de valorização social, graficamente, parece evidente na rede da Figura 2 que o fato de essa postulação ainda se encontrar pendente de concretização pouco interfere na trajetória de mudança institucional radical por que passou 
Mapa de Rede de Impactos para Gestão Estratégica na Universidade

a universidade. Nessa mesma perspectiva, podem-se incluir, por exemplo, os projetos Universidade Popular e Universidade Livre, que pouca ou nenhuma repercussão exerceriam sobre o conjunto de elementos da rede de impactos e seus conexos no sentido da transformação institucional.

Figura 2 - Mapa de Rede de Impactos no Processo de Planejamento da Renovação (2006-2010)

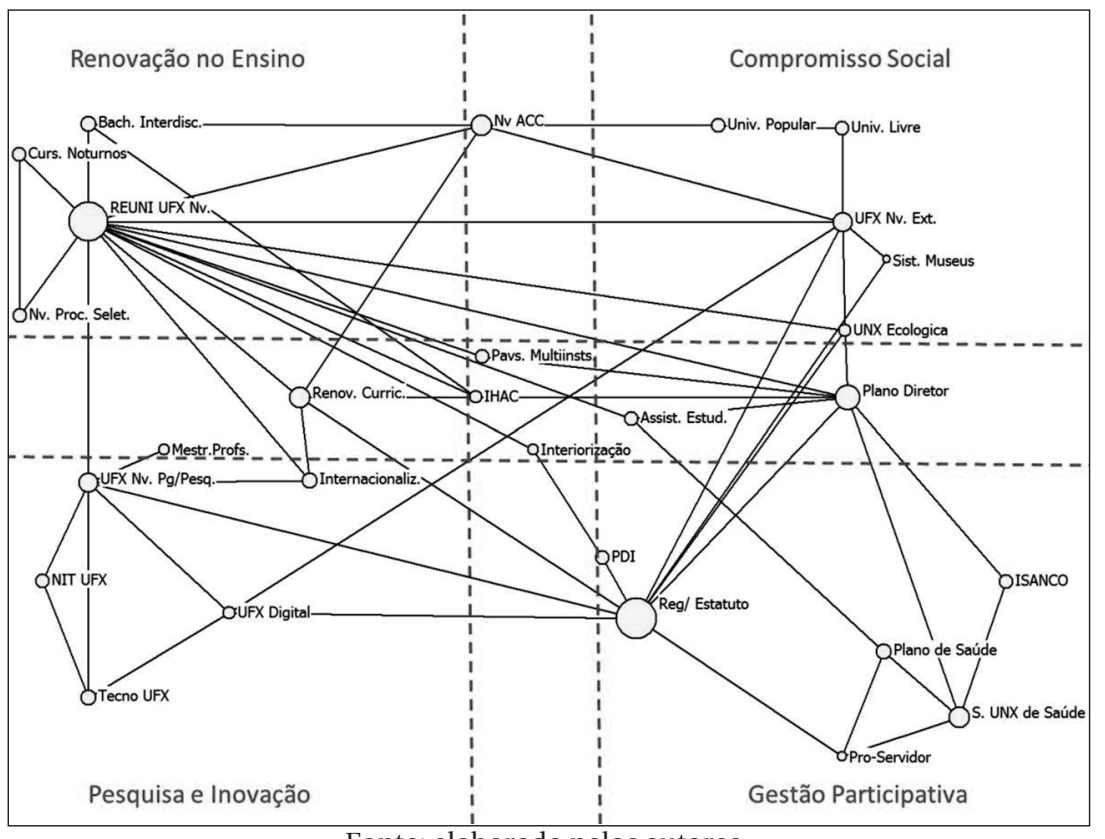

Fonte: elaborado pelos autores.

\section{Caracterização e Análise do Caso Estudado}

Como ilustração, o Quadro 2 sintetiza os resultados da incorporação de técnicas não lineares de gestão estratégica, mediante indicadores pertinentes no começo e no fim do período analisado no estudo de caso, destacando a variação percentual equivalente. O esquema permite articular as iniciativas apresentadas no Mapa de Rede de Impactos, possibilitando melhor entendimento da situação institucional da Universidade antes e depois da adoção da modalidade de planejamento estratégico considerada.

Como resultado, em 2010, a Universidade já matriculava um total de 32.412 estudantes, sendo 28.477 em cursos de graduação e 3.935 em cursos de pós-graduação. Isso significa um crescimento bruto de $65 \%$ em comparação com o ano-base. É importante assinalar que o crescimento bruto de matrículas foi maior na pós-graduação estrito senso 
Almeida Filho; Quintella; Coutinho; Mesquita; Barreto Filho

(135\% no período), atingindo marcas excepcionais (153\%) para o nível de doutorado.

Em 2010, quase 8,5 mil vagas foram abertas em 112 cursos de graduação. Em comparação com 2002, trata-se de um crescimento bruto de quase $120 \%$ na oferta de vagas. Mais de $1 / 3$ dessas vagas destinam-se a 40 cursos noturnos. Além de mais vagas nas licenciaturas, incluindo 450 lugares no Programa Especial de Formação Docente, abriramse quase 1,6 mil vagas em bacharelados interdisciplinares. Vinculado à implantação do Programa de Ações Afirmativas, pôde-se aumentar em mais de 60 vezes a oferta de vagas noturnas e passar de nenhuma para mais de mil bolsas de auxílio-permanência; tais iniciativas certamente contribuíram para o extraordinário aumento, da ordem de $470 \%$, da presença de estudantes pobres na Universidade.

Na pós-graduação estrito senso, a instituição contava em 2010 com 103 cursos - 61 mestrados e 42 doutorados -, ofertando quase duas mil vagas anuais. Para doutorado, são 643 vagas anuais, o que equivale a um aumento de $300 \%$ em relação a 2002 . De acordo com a avaliação trienal da Capes de $2009^{9}$, mais de $80 \%$ dos cursos haviam alcançado conceito 4 ou 5, resultando numa média geral de 4,2 (4,5 para os doutorados).

Em 2002, 30\% dos cursos de pós-graduação estrito senso tinham conceito 3 e nenhum dos programas tinha nota acima de 5. Hoje, apenas $18 \%$ dos cursos de pós-graduação têm nível 3. No outro extremo da escala, uma dezena de cursos foi classificada com conceito 6 ou mais (um deles em nível 7), ou seja em níveis de excepcionalidade, o que os coloca no seleto grupo dos considerados pela Capes como de nível internacional.

Em 2010, a Universidade contava com 530 grupos de pesquisa registrados no CNPq, resultado de um incremento de $137 \%$ em comparação com 2002. Do contingente de 2.203 pesquisadores ativos, 219 eram Bolsistas de Produtividade do CNPq (20 classificados no nível máximo 1-A); em 2002, havia apenas 106 pesquisadores bolsistas do CNPq, um crescimento de $100 \%$. Os dados indicam que, nos últimos anos, a produção científica cresceu em maior escala do que a produção brasileira. No período 2000 a 2003, a produção de artigos científicos indexados no ISI ${ }^{10}$ (Institute for Scientific Information) era de aproximadamente 1,2\% em relação à produção brasileira; esse percentual cresceu para quase $2 \%$ após 2008. Em 2009, os pesquisadores da instituição-caso publicaram um total de 536 artigos indexados na Web of Science, o que representa 0,19 artigos/docente. Em termos comparativos, a produção acadêmica quase duplicou em relação ao ano-base 2002.

Educação \& Realidade, Porto Alegre, v. 39, n. 1, p. 277-301, jan./mar. 2014.

Disponível em: <http://www.ufrgs.br/edu_realidade> 
Mapa de Rede de Impactos para Gestão Estratégica na Universidade

Quadro 2 - Indicadores Acadêmicos e Institucionais (2002-2010)

\begin{tabular}{|c|c|c|c|}
\hline Itens de avaliação & 2002 & 2010 & Var $\%$ \\
\hline Opções de cursos de graduação & 55 & 112 & 103,6 \\
\hline Vagas na graduação total & 3.851 & 8.446 & 119,3 \\
\hline Vagas na graduação em cursos noturnos & 40 & 2.610 & 6.425 \\
\hline Vagas na graduação em bacharelados interdisciplinares & 0 & 1.460 & $\infty$ \\
\hline Total de matrículas na graduação & 17.941 & 28.477 & 58,8 \\
\hline Cursos de pós-graduação & 61 & 103 & 68,8 \\
\hline Mestrados & 44 & 61 & 38,6 \\
\hline Doutorados & 17 & 42 & 147,1 \\
\hline Vagas na pós-graduação total & 749 & 1.896 & 153,1 \\
\hline Vagas na pós-graduação em mestrados & 590 & 1.253 & 112,4 \\
\hline Vagas na pós-graduação em doutorados & 159 & 643 & 304,4 \\
\hline Matrículas na pós-graduação & 1.672 & 3.935 & 135,3 \\
\hline Matrículas em mestrados & 1.057 & 2.380 & 125,2 \\
\hline Matrículas em doutorados & 615 & 1.555 & 152,9 \\
\hline $\begin{array}{l}\text { Estudantes de graduação de escolas públicas (critério } \\
\text { cotas) }\end{array}$ & 2.022 & 11.485 & 468,0 \\
\hline Convênios com universidades estrangeiras & 53 & 229 & 332,0 \\
\hline Estudantes da UFX em intercâmbio no exterior & 21 & 151 & 619,0 \\
\hline Grupos de pesquisa cadastrados no CNPq & 225 & 530 & 136,6 \\
\hline $\begin{array}{l}\text { Número de cursos de pós-graduação com conceito Capes } \\
6+\end{array}$ & 0 & 10 & $\infty$ \\
\hline Bolsistas de Produtividade CNPq & 106 & 219 & 106,6 \\
\hline Artigos publicados (Web of Science) & 198 & 536 & 170,7 \\
\hline Patentes requeridas/marcas registradas & 0 & 31 & $\infty$ \\
\hline Projetos Atividades Curriculares em Comunidade & 42 & 48 & 14,3 \\
\hline Total de participantes em projetos de Extensão & 19.509 & 25.758 & 32,0 \\
\hline Número de pavilhões de aulas & 2 & 9 & 125,0 \\
\hline Total de salas de aula & 286 & 594 & 107,7 \\
\hline Área total construída & 285.413 & 373.651 & 30,9 \\
\hline Acervo bibliográfico (livros + periódicos) & 259.342 & 716.936 & 176,4 \\
\hline Servidores técnicos e administrativos & 3.211 & 3.262 & 0,2 \\
\hline Número de docentes (quadro permanente) & 1.901 & 2.699 & 42,0 \\
\hline Proporção de doutores no quadro docente & $33,4 \%$ & $64,2 \%$ & 92,2 \\
\hline Razão aluno/professor (RAP)* & $11,3: 1$ & $16,4: 1$ & 45,1 \\
\hline
\end{tabular}

Legenda: $\infty$ - estimativa não mensurável; * RAP = Total alunos (graduação + PG) / Total professores equivalentes / 1,55

Fonte: elaborado pelos autores.

294 Educação \& Realidade, Porto Alegre, v. 39, n. 1, p. 277-301, jan./mar. 2014. Disponível em: <http://www.ufrgs.br/edu_realidade> 
Um dos indicadores mais robustos da qualidade de artigos publicados é o número de vezes que o trabalho é citado em outros textos científicos. De fato, observa-se um crescimento marcante do número de citações de artigos ao longo dos anos. Em 2002, 1.474 artigos científicos produzidos haviam sido citados em trabalhos indexados no ISI; em 2009, este número foi de 5.550, representando um incremento de $276 \%$.

No âmbito da Extensão, o programa Atividades Curriculares em Comunidade $^{11}$ (ACC) passou por profunda reformulação, ganhou estabilidade e experimentou uma pequena expansão. Dos 444 estudantes engajados em 42 grupos de ACC em 2002, passou-se a 48 grupos e 612 estudantes em 2009. No total, em 2009, foram computadas 647 ações extensionistas, como programas, projetos, ações, prestação de serviços ou eventos, mobilizando quase 26 mil participantes. Na comparação com 2002, verificamos que houve uma concentração de esforços, com redução do numero de projetos, simultânea à ampliação, em mais de $30 \%$, do universo de participantes. Na interface extensão-pesquisa, em 2010, o Núcleo de Inovação Tecnológica já computava mais de 30 requerimentos de patentes ou registro de marcas; em 2002, nenhum registro havia a declarar.

Em números consolidados, em 2010, a Universidade apresentou os seguintes dados físicos: área total de 532,42 hectares; sendo 77,02 ha na sede; 54,19 ha no Campus 1 do interior; 8,14 ha no Campus 2 e 393,07 ha em três fazendas experimentais. Nessa área própria, as benfeitorias totalizam 386 mil m² de área construída em 139 edificações, com um total de 594 instalações de ensino, incluindo salas e auditórios, concentrados em nove pavilhões de aulas, distribuídos em todos os campi. Em 2002, eram menos de 300 salas, distribuídas em 122 imóveis das unidades e órgãos e em apenas dois pavilhões de aula, com pouco mais de 250 mil $\mathrm{m}^{2}$ de área construída. Entre 2002 e 2010, foram incorporados ao patrimônio da instituição quase $140 \mathrm{mil} \mathrm{m}^{2}$ de área construída, ou 54\% de acréscimo.

Em 2007, o Conselho Universitário aprovou a criação do Sistema de Bibliotecas, coordenado por uma superintendência específica. O efeito imediato foi maior racionalidade na gestão, manutenção e operação do acervo, além de ampliação dos horários de funcionamento, alternativos às aulas. Em 2010, o acervo conjunto compreendia 673.599 títulos de livros (eram 247.547 em 2002), além de 17.428 periódicos nacionais e estrangeiros; a taxa correspondente de aumento do acervo total corresponde a quase $180 \%$ no período.

Em agosto de 2010, o quadro docente compunha-se de 2.699 professores, sendo 1.992 professores do quadro permanente e 689 substitutos, correspondendo a 3.055 unidades de professor equivalente ${ }^{12}$. Em relação a 2002, trata-se de uma ampliação de $17 \%$ do total de professores. Somente no ano de 2009, no contexto do Reuni, foram contratados 244 docentes. Em 2010, 68\% dos docentes eram doutores; em 2002, os

Educação \& Realidade, Porto Alegre, v. 39, n. 1, p. 277-301, jan./mar. 2014.

Disponível em: <http://www.ufrgs.br/edu_realidade> 
Mapa de Rede de Impactos para Gestão Estratégica na Universidade

doutores eram apenas $38 \%$ do quadro docente. O corpo técnico-administrativo totalizava 3.262 servidores, com $27 \%$ de nível superior.

\section{Discussão}

O presente estudo de caso, enfim, avalia contexto, programação e resultados comparativamente em duas etapas: Fase 1 - empregando abordagens de planejamento linear (2002-2006) versus Fase 2 - com adoção de um modelo de planejamento não linear (2006-2010). Diagnóstico detalhado de crises e contexto institucional, tratamento cuidadoso de situações-problema, saneamento financeiro, reorganização administrativa, crescimento na graduação e na pós-graduação, lançamento de alguns programas estratégicos de mudança, tudo isso fez da Fase 1 etapa preparatória para avanços e propostas de maior fôlego e ambição. A Fase 2 justificou-se essencialmente como sequência, oportuna e necessária, de um projeto político-acadêmico que almejou profunda e radical transformação institucional. Com esse sentido, a equipe dirigente reafirmou princípios, diretrizes, estratégias, propostas e metas que já constavam de documentos produzidos pelas equipes de concepção, planejamento e gestão. Dado o caráter de renovação organizacional e acadêmica implicado no programa de trabalho realizado, foram superados vetores institucionais de inércia, conservação e permanência. Buscou-se enfim aplicar métodos, técnicas e instrumentos de planejamento orientado à mudança e de gestão da inovação consistentes com o horizonte de transformação almejado.

Superando décadas de estagnação que, em 2002, resultaram num contingente de estudantes quantitativamente desproporcional à expressão da instituição e à demanda da sociedade, a Universidade experimentou vigoroso crescimento. Em oito anos, o aumento de cursos e vagas de graduação foi quase $120 \%$, ultrapassando a marca de $30 \mathrm{mil}$ matrículas em 113 cursos no ano de 2010. Em relação à pós-graduação e à pesquisa, os patamares de ampliação efetivamente alcançados foram ainda mais expressivos, variando de até $150 \%$ para vagas e matrículas a mais de $170 \%$ nos indicadores de produtividade em pesquisa. Nesse aspecto, o maior destaque foi para o aumento de $300 \%$ na oferta de vagas em doutoramento.

Aumentos excepcionais em todos os indicadores de internacionalização (em comparação com 2002: 330\% em número de convênios, $600 \%$ de aumento na presença de seus estudantes em universidades estrangeiras e aumento de $340 \%$ de estudantes internacionais na instituição) atestam essa faceta da mudança radical da Universidade. Para avaliar as condições institucionais que resultaram do processo de renovação da instituição, consideremos dois aspectos: estrutura física, incluindo infraestrutura dos campi, edificações e instalações de uso administrativo e acadêmico, e quadro de pessoal, em termos quantitativos e qualitativos. A operação dessa complexa estrutura institucional 
visando às atividades-fim da Universidade demanda quadros docentes, técnicos e administrativos em número suficiente, com alta qualificação e competência. Em quase todos esses aspectos (à exceção dos servidores administrativos), a evolução positiva dos indicadores é flagrante no período considerado no estudo de caso.

Evidentemente, porém, não se pode atribuir os avanços acontecidos na Universidade exclusivamente ao seu planejamento não linear ou, em particular, ao Mapa de Rede de Impactos. Diversos fatores externos de enorme importância concorreram para que tais resultados fossem alcançados, valendo destacar principalmente o sensível aumento do volume de recursos disponibilizados pelo Governo Federal no período. Assim, buscou-se, aqui, divulgar a ferramenta e traçar uma breve comparação de fases (com e sem planejamento não linear). Não obstante, de fato, a utilização do modelo MRI foi fundamental para o sucesso da gestão, particularmente por proporcionar uma visão global e interativa dos inúmeros projetos conduzidos entre 2006-2010. A ferramenta mostrouse, também, utilíssima na priorização sistêmica de ações e programas, processo bastante distinto da priorização individualizada de projetos, típica do planejamento tradicional. Entende-se, portanto, que o presente artigo contribui com o avanço do campo da gestão estratégica ao preencher a lacuna existente em termos de estudos sobre a aplicação de Mapas de Redes de Impacto em organizações hipercomplexas.

O desenho metodológico deste estudo de caso é do tipo diacrônico, pois contrasta momentos sucessivos de desenvolvimento de uma mesma instituição, diferenciados pela metodologia de gestão estratégica empregada. Outra possibilidade de grande potencial para avaliar eficácia e efetividade de abordagens de planejamento não linear em organizações hipercomplexas da modalidade universitária será a abordagem sincrônica. Nesse sentido, recomendam-se novos estudos, com a finalidade de comparar situações, processos e indicadores de resultados entre instituições que, submetidas a um conjunto similar de condições e investimentos (como o Programa Reuni, por exemplo), utilizaram modelos alternativos de gestão estratégica para os seus respectivos planos de reestruturação.

Recebido em 17 de setembro de 2012 Aprovado em 11 de maio de 2013

\section{Notas}

1 A perspectiva porteriana é aquela que se origina no campo da Organização Industrial. Foi o paradigma dominante do pensamento estratégico nos anos 1980 e 1990. A partir dos anos 2000, a Visão Baseada em Recursos (Barney, 2001) ganha força como paradigma alternativo.

2 O conceito de gestão estratégica de Igor Ansoff (1976) baseia-se na percepção de que o planejamento é apenas a primeira etapa de um processo muito mais complexo, aquele que visa a tornar as decisões cotidianas das organizações alinhadas aos seus respectivos planejamentos estratégicos. 
Mapa de Rede de Impactos para Gestão Estratégica na Universidade

3 Tabatoni, Davies e Barblan (2009) observam que a European Universities Association reúne hoje 650 associados de 45 distintos países.

4 Por várias razões, percebe-se que a função do conhecimento na universidade está sendo minada. Alguns, de fato, chegam a sugerir que estamos testemunhando "o fim do conhecimento" na educação superior (tradução nossa).

5 Disponível em: <http://networkingaction.net/2010/03/strategic-mapping-fornetworks>. Acesso em: 2 set. 2012.

6 O BSC ou Balanced Scorecard (Kaplan; Norton, 1992) é uma ferramenta de gestão estratégica que visa, a partir de um elenco de perspectivas e indicadores, a assegurar que as organizações consigam manter a coerência entre a gestão de suas rotinas e seu planejamento estratégico.

7 Os mapas estratégicos (Kaplan; Norton, 2004) são diagramas de causa e efeito que mostram como a associação dos objetivos estratégicos de cada perspectiva organizacional pode levar as organizações a alcançar suas visões de futuro.

8 "As universidades devem exercer sua autonomia institucional para propor cursos novos, flexibilidade curricular, caminhos de formação adaptados a cada realidade local. Ao se evitar a especialização precoce, ditada por uma formação estritamente profissionalizante, torna-se possível utilizar, de forma mais eficiente, os recursos humanos e materiais existentes" (Diretrizes Gerais para o Reuni, MEC, 2007, p. 22. Disponível em: <http://portal.mec.gov.br/sesu/ arquivos/pdf/diretrizesreuni.pdf $>$. Acesso em: 26 jun. 2012).

9 Disponível em: <http://trienal.capes.gov.br>. Acesso em: 26 jun. 2012.

10 Disponível em: <http://isiknowledge.com>. Acesso em: 2 set. 2012.

11 Disponível em: <http://www.acc.ufba.br>. Acesso em: 2 set. 2012.

12 Medida segundo a qual "[...] as universidades podem contratar, por meio de concurso público e nos limites fixados para cada instituição, professores efetivos, substitutos e visitantes, sem depender de autorização específica dos Ministérios da Educação e Planejamento, Orçamento e Gestão. De acordo com o Decreto n ${ }^{\circ}$ 7.485, de 18 de maio, a referência para cada professor equivalente é o professor de terceiro grau, classe adjunto, nível 1, com regime de trabalho de quarenta horas semanais e titulação equivalente a doutor" (Disponível em: <http:// portal.mec.gov.br/index.php?option=com_content\&view=article\&id=16638>. Acesso em: 7 set. 2012).

\section{Referências}

ANSOFF, Igor. From Strategic Planning to Strategic Management. London: John Wiley, 1976.

ANSOFF, Igor. Critique of Henry Mintzberg's 'The Design School; Reconsidering the Basic Premises of Strategic Management'. Strategic Management Journal, West Lafayette, v. 12, n. 6, p. 449-461, 1991.

BARNETT, Ronald. University Knowledge in an Age of Supercomplexity. Higher Education, Finland, v. 40, n. 4, p. 409-422, 2000.

BARNEY, Jay B. Is the Resource-Based Theory a Useful Perspective for Strategic Management Research? Yes. Academy of Management Review, New York, v. 26, n. 1, p. 41-56, 2001.

CAO, Zhongqiu; ZHANG, Xinmin. On the Strategic Management of the Colleges and Universities. 2009. Disponível em: <http://www.seiofbluemountain.com/ upload/product/200909/2009jyhy02a31.pdf>. Acesso em: 26 jun. 2012. 
CYERT, Richard; MARCH, James. A Behavioral Theory of the Firm. New Jersey: Prentice Hall, 1963.

DUKE, Chris. The Learning University: towards a new paradigm? Buckingham [England]; Philadelphia: Society for Research into Higher Education \& Open University Press, 1992.

EARL, Sarah; CARDEN, Fred; SMUTYLO, Terry. Outcome Mapping: building learning and reflection into development programs. Ottawa: International Development Research Centre (IDRC), 2001.

HAMMER, Michael. Deep Change: how operational innovation can transform your company. Harvard Business Review, Cambridge (EUA), 1 abr. 2004.

KAPLAN, Robert; NORTON, David P. The Balanced Scorecard: measures that drive performance. Harvard Business Review, Cambridge (EUA), jan./fev. 1992. KAPLAN, Robert; NORTON, David P. Strategy Maps: converting intangible assets into tangible outcomes. Cambridge (EUA): Harvard Business School Press, 2004.

KELLER, George. Academic Strategy: the management revolution in American Higher Education. Baltimore: Johns Hopkins University Press, 1983.

KIEL, L. Douglas. Managing Chaos and Complexity in Government: a new paradigm for managing change, innovation and organizational renewal. San Francisco: Jossey-Bass, 1994.

KIEL, L. Douglas (Org.). Knowledge Management, Organizational Intelligence and Learning, and Complexity. Paris: Unesco (EOLSS), 2008.

LEÃO, Izabel. Engenheiros conectados com o social. Jornal da USP, São Paulo, v. 18, n. 654, 18-24 ago. 2003. Disponível em: <http://www.usp.br/jorusp/arquivo/2003/jusp654/pag09.htm>. Acesso em: 1 set. 2007.

LERNER, Alexandra L. A Strategic Planning Primer for Higher Education. Northridge (EUA): College of Business Administration and Economics, California State University, 1999.

MINTZBERG, Henry. The Design School: reconsidering the basic premises of strategic management. Strategic Management Journal, West Lafayette, v. 11, n. 3, p. 171-195, mar./abr. 1990.

MINTZBERG, Henry. That's Not Turbulence, Chicken Little, It's Real Opportunity. Planning Review, nov./dez. 1994.

MINTZBERG, Henry; AHLSTRAND, Bruce; LAMPEL, Joseph. Strategy Safari: a guided tour through the wilds of strategics management. New York: The Free Press, 1999.

MUNCK, Ronaldo; MCCONNELL, Gordon. University Strategic Planning and the Foresight/Futures Approach: an Irish case study. Planning for Higher Education, Ann Arbor, v. 38, n. 1, p. 31-40, out./dez. 2009.

NIVEN, Paul R. Balanced Scorecard Step-By-Step: maximizing performance and maintaining results. New Jersey: John Wiley \& Sons, 2008.

PARIS, Kathleen A. Strategic Planning in the University. Madison: University of Wisconsin System Board of Regents, 2003. Disponível em: <http://oqi.wisc.edu/ resourcelibrary/uploads/resources/Strategic Planning in the University.pdf $>$. Acesso em: 26 jun. 2012.

PATTON, Michael Quinn. Developmental Evaluation. Evaluation Practice, Minneapolis, v. 15, n. 3, p. 311-319, 1994. 
Mapa de Rede de Impactos para Gestão Estratégica na Universidade

PATTON, Michael Quinn. Development Innovation: applying complexity concepts to enhance innovation and use. New York: Guilford Press, 2010.

PORTER, Michael. Competitive Advantage: techniques for analyzing industries and competitors. New York: Free Press, 1998.

PRAHALAD, Coimbatore Krishnarao; HAMEL, Gary. The Core Competence of the Corporation. Harvard Business Review, Cambridge (EUA), v. 68, n. 3, p. 7991, maio 1990

QUINN, Robert. Deep Change: Discovering The Leader Within. San Francisco: Jossey-Bass, 1996.

QUINTELLA, Rogério H. The Strategic Management of Technology in the Chemical and Petrochemical Industries. London: Pinter Publishers, 1993.

QUINTELLA, Rogério H.; CABRAL, Sandro. Um Modelo Espacial para Análise e Ensino de Escolas de Pensamento Estratégico. Revista Brasileira de Administração Pública, Rio de Janeiro, v. 41, p. 1165-1188, 2007. Disponível em: <http:// www.scielo.br/scielo.php?script=sci_arttext\&pid=s0034-76122007000600008>. Acesso em: 16 ago. 2010.

RAYASAM, Renuka. Why Workplace Democracy Can Be Good Business. U.S. NEWS \& WORLD REPORT. 2008. Disponível em: <http://money.usnews.com/ money/careers/articles/2008/04/24/why-workplace-democracy-can-be-goodbusiness $>$. Acesso em: 16 ago. 2010.

ROWLEY, Daniel J.; LUJAN, Herman D.; DOLENCE, Michael G. Strategic Change in Colleges and Universities. San Francisco: Jossey-Bass Publishers, 1997.

SENGE, Peter. A Quinta Disciplina: arte e prática da organização que aprende. Rio de Janeiro: Best Seller, 2010.

SENGE, Peter. Creating the Schools of the Future: Education for a Sustainable Society. Solutions Journal, Burlington, v. 3, n. 3, jun. 2012. Disponível em: <http://www.thesolutionsjournal.com/node/1116>. Acesso em: 21 abr. 2013.

SENGE, Peter et al. Presence: an exploration of profound change in people, organizations, and society. New York: Doubleday, 2005.

SMUTYLO, Terry. Outcome Mapping: a method for tracking behavioural changes in development programs. Roma: Institutional Learning and Change (ILAC) Initiative, ILAC Brief, n. 7, 2005.

TABATONI, Pierre; DAVIES, John; BARBLAN, Andris. Strategic Management and Universities' Institutional Development. 2009. Disponível em: <http:// www.eua.be/eua/jsp/en/upload/Strategic_Manag_Uni_institutional_Devlpt.1069322397877.pdf>. Acesso em: 27 dez. 2013.

TAVERNIER, Karel. Relevance of Strategic Management for Universities. Tijdschrifr voor Economie en Management, Leuven, v. 1, n. 5, 2005. Disponível em: <https://lirias.kuleuven.be/bitstream/123456789/120121/1/TEM_5_05_Tavernier.pdf $>$. Acesso em: 26 jun. 2012.

VASCONCELOS, Flávio. Safári de Estratégia, Questões Bizantinas e a Síndrome do Ornitorrinco: uma análise empírica dos impactos da diversidade teórica em estratégia empresarial sobre a prática dos processos de tomada de decisão estratégica. In: ENCONTRO DA ANPAD, 25, 2001, Campinas. Anais... Campinas: ANPAD, 2001, 1CD.

WADDELL, Steve. Strategic Mapping for Networks. 2010. Disponível em: $<$ http:// networkingaction.net/2010/03/strategic-mapping-for-networks $>$. Acesso em: 6 ago. 2011. 
Naomar Monteiro de Almeida Filho é professor titular do Instituto de Saúde Coletiva da UFBA. Reitor pro tempore da Universidade Federal do Sul da Bahia, Bolsista de Produtividade 1A do CNPq. PhD em Epidemiologia pela Universidade da Carolina do Norte (EUA).

E-mail: naomaralmeida@gmail.com

Rogério Hermida Quintella é professor titular e pró-reitor pro tempore de Sustentabilidade e Integração Social da Universidade Federal do Sul da Bahia. Bolsista de Produtividade $1 \mathrm{C}$ do CNPq e membro do Comitê de Assessoramento de Administração deste Conselho. PhD em Gerenciamento Estratégico de Tecnologia pela University of Brighton.

E-mail: rhquintella@pq.cnpq.br

Denise Maria Barreto Coutinho é professora adjunta do Instituto de Psicologia da UFBA. Docente permanente do Programa de Pós-Graduação em Artes Cênicas e colaboradora do PPG em Psicologia, ambos da UFBA. Bolsista de Pós-Doutorado da FAPESB 2013-2014. Doutora em Letras pela UFBA/Princeton University (EUA).

E-mail: denisecoutinhol@gmail.com

Francisco José Gomes Mesquita é pró-reitor pro tempore de Administração e Planejamento da Universidade Federal do Sul da Bahia. Professor adjunto do Instituto de Geociências da Universidade Federal da Bahia. Mestre e doutorando em Geociências pela Universidade Federal da Bahia. Foi vicereitor da UFBA.

E-mail: fmesquita@ufba.br

Osvaldo Barreto Filho é secretário de Educação do Estado da Bahia. Exdiretor da Escola de Administração e Professor Adjunto da UFBA. Graduado em Economia, especialista em Planejamento e mestre em Administração pela UFBA.

E-mail: bfosvaldo@gmail.com 